coefficient of PTH $(\beta=0.199$ to $\beta=0.213,7.0 \%)$ and was not a significant predictor of CIMT $(p=0.209)$, suggesting that the relationship was not mediated by vitamin $\mathrm{D}$.

Conclusion In this high-risk older cohort, high serum PTH levels are associated with increased CIMT independent of traditional atherosclerotic risk factors.

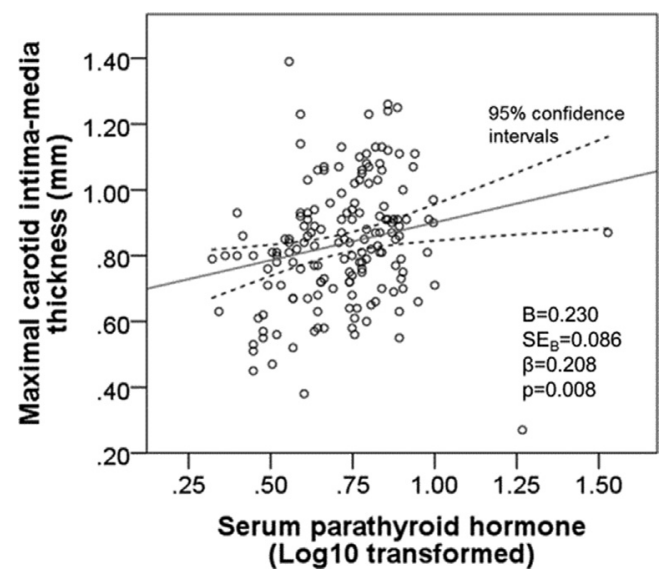

Abstract 56 Figure 1

\section{SERUM TOTAL VITAMIN D LEVELS ARE NOT ASSOCIATED WITH ENDOTHELIAL DYSFUNCTION, VASCULAR STIFFNESS OR EARLY ADVERSE OUTCOMES AFTER INVASIVE MANAGEMENT OF NON-ST ELEVATION ACUTE CORONARY SYNDROME IN OLDER PATIENTS}

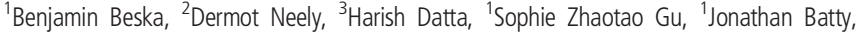
${ }^{4}$ Hannah Sinclair, ${ }^{5}$ Guy MacGowan, ${ }^{6}$ Gary Ford, ${ }^{7}$ Weiliang Qiu, ${ }^{1}$ Vijay Kunadian*. ${ }^{1}$ Institute of Cellular Medicine, Faculty of Medical Sciences, Newcastle University; ${ }^{2}$ Department of Biochemistry, Newcastle upon Tyne Hospitals NHS Foundation Trust; ${ }^{3}$ Institute of Cellular Medicine, Newcastle University, and Department of Biochemistry, Newcastle upon Tyne Hospitals NHS Foundation Trust; ${ }^{4}$ Institute of Cellular Medicine, Newcastle University, and Cardiothoracic Centre, Freeman Hospital; ${ }^{5}$ Cardiothoracic Centre, Freeman Hospital, Newcastle upon Tyne Hospitals NHS Foundation Trust; ${ }^{6}$ Divison of Medical Sciences, Oxford University; ${ }^{7}$ Channing Division of Network Medicine, Department of Medicine, Brigham and Women's Hospital/Harvard Medical School
\end{abstract}

\subsection{6/heartjnl-2017-311726.56}

Introduction Vitamin D may be an important biomarker of cardiovascular disease. Older patients are at particularly high risk of adverse outcomes following non-ST elevation acute coronary syndrome (NSTEACS). Low vitamin D has been previously linked to endothelial dysfunction and vascular stiffness. Therefore, the higher incidence of low vitamin $\mathrm{D}$ in older adults may play a plausible mechanistic role in predisposing this cohort to higher risk. The association between vitamin D, endothelial function, vascular stiffness and early outcomes after invasive management of NSTEACS in older patients was evaluated.

Methods We evaluated 294 consecutive older patients (aged 65 years) with NSTEACS managed by an invasive strategy. Serum total vitamin D was measured pre-procedure by electrochemiluminescent immunoassay. Endothelial function was determined using peripheral arterial tonometry and vascular stiffness with carotid-femoral pulse wave velocity (PWV), both within 24 hours post-procedure. Major Adverse Cardiovascular Events (MACE) were defined as 30 day composite of all-cause mortality, acute coronary syndrome, unplanned repeat revascularisation, significant bleeding, stroke or transient ischaemic attack. Multivariate linear and logistic regressions were performed controlling for age, sex, serum triglycerides, hypertension, diabetes and smoking status.

Results Mean age was $80.5 \pm 4.8$ years (61.9\% male). Median vitamin D level was $29.5 \mathrm{nmol} / \mathrm{L}$ [IQR $16.0-53.0 \mathrm{nmol} / \mathrm{L}]$ and was split into quartiles for analysis: Q1 $(16.0 \mathrm{nmol} / \mathrm{L}, \mathrm{n}=72)$, Q2 (17.0-29.5 nmol/L, $\mathrm{n}=75)$, Q3 (30.0-53.0 nmol/L, $\mathrm{n}=72$ ) and Q4 $(54.0 \mathrm{nmol} / \mathrm{L}, \mathrm{n}=72)$. There was no difference in endothelial function $(p=0.337)$ or PWV $(p=0.633)$ between the vitamin $\mathrm{D}$ quartiles. There was no adjusted linear relationship between vitamin D and PWV $(p=0.410)$ or endothelial function $(\mathrm{p}=0.490)$. MACE incidence did not differ between quartiles (Q1 2.7\%; Q2 0\%; Q3 5.3\%; Q4 5.6\%; p=0.210) and adjusted for confounders, did not significantly predict MACE $(p=0.083)$.

Conclusion In this high-risk older cohort, pre-procedural serum total vitamin D level is not associated with endothelial dysfunction or vascular stiffness and is not predictive of shortterm outcomes after invasive management of NSTEACS.

\section{OPTIMAL RISK STRATIFICATION PATHWAYS FOR PATIENTS WITH SUSPECTED ACUTE CORONARY SYNDROME}

${ }^{1}$ Andrew Chapman*, ${ }^{2}$ Atul Anand, ${ }^{2}$ Dennis Sandeman, ${ }^{1}$ Amy Ferry, ${ }^{2}$ Anoop Shah, ${ }^{1}$ Philip Adamson, ${ }^{1}$ Fiona Strachan, ${ }^{1}$ Alasdair Gray, ${ }^{1}$ David Newby, ${ }^{1}$ Nick Mills. ${ }^{1}$ University of Edinburgh; ${ }^{2}$

\subsection{6/heartjnl-2017-311726.57}

Background Novel pathways utilise low concentrations of cardiac troponin and a normal ECG to risk stratify patients with suspected acute coronary syndrome. However, clinical risk scores incorporating additional cardiovascular risk factors or physiological parameters are commonly used in practice. Whether such clinical risk scores are safer than novel pathways is uncertain.

Methods Patients with suspected acute coronary syndrome $(\mathrm{n}=1,139)$ underwent high-sensitivity cardiac troponin I testing at presentation, 3 and 6 or 12 hours. We applied the HighSTEACS pathway, which rules out myocardial infarction in those without ischaemia on the ECG if troponin concentrations are $<5 \mathrm{ng} / \mathrm{L}$ at presentation and symptom duration is 2 hours. Early presenters and those $5 \mathrm{ng} / \mathrm{L}$ are ruled out if absolute change is $<3 \mathrm{ng} / \mathrm{L}$ at 3 hours and they remain 99th centile. We compared the HighSTEACS pathway with the HEART score (low risk 3), or a TIMI score of 0 with a normal ECG. We compared the negative predictive value (NPV) and efficacy of each approach for a primary outcome of index type 1 myocardial infarction, or type 1 myocardial infarction or cardiac death at 30 days.

Results The primary outcome occurred in $15.5 \%(177 / 1,139)$. The HighSTEACS pathway ruled out $61.9 \%(705 / 1,139)$ of patients by three hours, with a NPV of $99.6 \%$ [95\%CI 99.1\%99.9\%]. The NPV of both the HEART and the TIMI score was similar (Figure 1A), however, they identified a significantly lower proportion of patients as eligible for discharge (HEART 18\% (205/1,139), TIMI 26.3\% (299/1,139); Figure 1B).

Conclusions The HighSTEACS pathway identifies patients at very low risk of index myocardial infarction, or myocardial infarction or cardiac death at 30 days, and rules out acute coronary syndrome in over half of patients presenting to the Emergency Department. Whilst the HEART and the TIMI 